\title{
The ears of the African elephant: unexpected high seroprevalence of Plasmodium ovale and Plasmodium malariae in healthy populations in Western Africa
}

\author{
Cécile Doderer-Lang ${ }^{1 *}$, Pascal S Atchade ${ }^{2}$, Lydia Meckert $^{1}$, Elodie Haar ${ }^{1}$, Sylvie Perrotey ${ }^{1}$, Denis Filisetti ${ }^{1}$, \\ Ahmed Aboubacar ${ }^{1}$, Alexander W Pfaff ${ }^{1}$, Julie Brunet ${ }^{1}$, Nicodème W Chabi ${ }^{2}$, Casimir D Akpovi ${ }^{2}$, Ludovic Anani ${ }^{3}$, \\ André Bigot ${ }^{3}$, Ambaliou Sanni ${ }^{2}$ and Ermanno Candolfi ${ }^{*}$
}

\begin{abstract}
Background: Malaria Is A Life-Threatening Pathology In Africa. Plasmodium Falciparum And Plasmodium Vivax Attract The Most Focus Because Of Their High Prevalence And Mortality. Knowledge About The Prevalence Of The Cryptic Pathogens Plasmodium Ovale And Plasmodium Malariae Is Limited. Thanks To Recombinant Tools, Their Seroprevalence Was Measured For The First Time, As Well As The Prevalence Of Mixed Infections In A Malaria-Asymptomatic Population In Benin, A Malaria-Endemic Country.
\end{abstract}

Methods: A Panel Of 1,235 Blood Donations Collected Over Ten Months In Benin Was Used For Validation Of The Recombinant Tools. Recombinant P. Falciparum, P. Malariae, P. Ovale MSP1, And P. Falciparum AMA1 Were Engineered And Validated On A Biobank With Malaria-Infected Patients $(N=144)$ Using A Species-Speific ELISA Test (Recelisa). Results Were Compared To An ELISA Using A Native P. Falciparum Antigen (NatELISA).

Results: Among Microscopically Negative African Blood Donors, $85 \%(1,050 / 1,235)$ Present Antibodies Directed To Native P. Falciparum, $94.4 \%(1,166 / 1,235)$ To rPfMSP1 And rPfAMA1, 56.8\% $(702 / 1,235)$ To rPoMSP1, 67.5\% (834/1235) To rPmMSP1 And 45.3\% Of The Malaria Seropositive Population Had Antibodies Recognizing The Three Species.

Conclusion: A High Rate Of Antibodies Against P. Ovale And P. Malariae Was Found In Asymptomatic Blood Donors. The Proportion Of Mixed Infections Involving Three Species Was Also Unexpected. These Data Suggest That Determining Seroprevalence For These Cryptic Species Is An Appropriate Tool To Estimate Their Incidence, At The Eve Of Upcoming Anti-P. Falciparum Vaccination Campaigns.

Keywords: Plasmodium, Seroprevalence, Recombinant protein, ELISA, Africa

\section{Background}

Malaria is one of the most life-threatening diseases in the world. In 2010, 219 million cases were reported globally [1], leading to 660,000 deaths, mostly of children under five years old. Five parasitic species cause human malaria (Plasmodium falciparum, Plasmodium vivax, Plasmodium malariae, Plasmodium ovale, and Plasmodium knowlesi),

\footnotetext{
*Correspondence: cecile.lang@unistra.fr; candolfi@unistra.fr

'Institut de Parasitologie et de Pathologie Tropicale Université de Strasbourg, 3, Rue Koeberlé, F67000 Strasbourg, France

Full list of author information is available at the end of the article
}

which are transmitted by the bite of infected Anopheles mosquitoes. Despite huge efforts to control the disease, resurgence has been observed in many countries due to climate instability, global warming, civil disturbances, drug resistance, and increasing travel between endemic and non-endemic areas [2].

Identifying the most affected countries' direct resources and validating control measures is essential to reducing malaria's incidence (target: $75 \%$ by 2015) [1]. Epidemiological surveillance seeks to assess malaria's prevalence over time and identify the species' geographical distribution. 
Vaccines against $P$. falciparum and $P$. vivax are in progress [3]; not so for P. malariae and P. ovale, where prevalence has been insufficiently investigated. To further complicate matters, low levels of parasitaemia and mixed infections can affect diagnosis of P. malariae and P. ovale. Birkenmeyer et al. recently sequenced genes encoding major erythrocyte stage markers of $P$. malariae and $P$. ovale, demonstrating the feasibility of Merozoite Surface Protein 1 (MSP1) recombinant production [4] and use in ELISA to detect antibodies against $P$. malariae and $P$. ovale [5]. Proteins were selected according to their ability to elicit antibody production and chosen from among previously described vaccine candidates. MSP1 is one of the main surface proteins at the merozoite stage expressed in all Plasmodium species and plays a role in red blood cell (RBC) invasions [6]. Plasmodium falciparum AMA1 is a blood-stage antigen that aids in orienting the merozoite during invasion of RBC. AntiAMA1 antibodies tend to be present in individuals who have acquired natural immunity [7].

Estimation of malaria prevalence is historically done by optical microscopy but a sensitivity of 50 parasites $/ \mu \mathrm{L}$ is insufficient [8]. Further, highly trained staff is necessary, rendering this approach unsuitable for large-scale monitoring. Rapid diagnostic tests and PCR methods are also inappropriate for broad evaluation. ELISA antigen detection of Plasmodium lactate dehydrogenase (pLDH) has been documented as a valuable tool for assessing prevalence in a blood donor population [9]. However, detectability is limited to one parasite $/ \mu \mathrm{L}$ and the assay is inappropriate for $P$. malariae and $P$. ovale identification. Furthermore, various factors influence the direct detection of parasites, among them parasite clearance due to acquired immunity, drug treatment, season variability and sporadic transmission in low-transmission areas. For this reason, seroprevalence measurement has been explored as an accurate tool for estimating transmission intensity and the potential effects of any measures used to control (and ultimately eliminate) malaria [10]. Indeed, antibodies against the four Plasmodium species appear within days or weeks of erythrocyte invasion, and can persist for months or years reflecting exposure to the parasites [11].

Immunofluorescence detection of malaria antibodies was until recently the gold standard [12], but is unsuitable for high-throughput screening. ELISA-based seroprevalence screening is a potentially useful epidemiological tool [13]. An immuno-enzymatic assay combining the crude $P$. falciparum antigen and recombinant $P$. vivax proteins was already developed, exhibiting high specificity and analytical sensitivity (96.7 and $93.1 \%$, respectively) in the detection of Plasmodium antibodies [14]. However, this technique could not discriminate between the four species.
In this work, the identification and production of recombinant proteins from $P$. falciparum, $P$. ovale and $P$. malariae was reported to establish an ELISA test for the detection of Plasmodium species-specific antibodies. Immunoassay performances were first assessed in a population of Plasmodium-infected French travellers. For the first time, the distribution of the $P$. malariae and $P$. ovale in endemic malaria areas in Benin (Western Africa) was evaluated in a blood donor population.

\section{Methods}

Samples from Plasmodium-infected patients for sensitivity calculation

Malaria patients returning from endemic countries sera $(n=144)$, diagnosed and referred at the Strasbourg University Hospital were used for the study. All patients reported fever and were microscopically diagnosed positive for P. falciparum $(\mathrm{n}=106)$, P. malariae $(\mathrm{n}=12)$, or $P$. ovale $(\mathrm{n}=26)$. All results were confirmed by PCR. Every patient was treated and the samples anonymized. This population was used to determine the recombinant ELISA assay's clinical sensitivity and positive predictive value.

\section{Negative samples}

Blood donor samples were collected at the Etablissement Français du Sang d'Alsace (EFS Alsace). Donors were classified as unexposed-to-malaria (192 samples) if their questionnaire responses indicated never having travelled to an endemic area. These samples were used to calculate the test's specificity and negative predictive value.

\section{Samples from Beninese blood donors}

Plasma and total blood samples from blood donors without apparent malaria symptoms $(n=1,235)$ were collected over ten months (May 2009 to February 2010) in six Beninese departmental blood centres [9]. Each donor signed a consent form, and both the Direction of Benin National Blood Transfusion Agency and the Research Ethics Committee of the Republic of Benin validated the protocol. The collection period was divided into a long rainy season (LRS) from May to July $(\mathrm{n}=387)$, a short dry season (SDS) from August to September $(n=217)$, a short rainy season (SRS) from October to November $(\mathrm{n}=408)$, and a long dry season (LDS) from December to February $(n=223)$. Two expert biologists performed parasitic examinations on all samples via microscopy on a May-Grünwald-Giemsa-stained thin and thick film. Positivity for Plasmodium was detected microscopically in 290 donors, who were used to assess the performance of the recombinant proteins. ELISA tests using a native $P$. falciparum antigen and recombinant proteins from $P$. falciparum, $P$. ovale and $P$. malariae were used to assess seroprevalence in the Beninese samples. Five donors 
gave their blood twice in two different seasons (LRS and SRS); they were kept due to the low number of repeat donors, since they were positive for malaria antibodies.

\section{Recombinant proteins}

Nucleotide constructs encoding 373 AA (amino acids) of P. falciparum MSP1 (Accession Number XP_001352170.1), 356 AA of $P$. ovale MSP1 (ACZ51239.1), and 350 AA of P. malariae MSP1 (ACZ51237.1) corresponding to the C-terminus region of the protein $(42 \mathrm{kD})$ and a nucleotide construct encoding 448 AA of $P$. falciparum AMA1 (XP_001348015.1) were commercially synthesized with an Escherichia coli codon bias (Genscript, Piscataway, NJ, USA). Consensus sequences derived from the alignment of several strains for each species and each protein were used for the design of synthetic genes. All genes were inserted in an expression vector containing a Maltose binding protein fusion partner pMAL-c2X ${ }^{\circ}$ (New England BioLabs, Ipswich, MA, USA), produced in E. coli expression hosts and purified on amylose resin and DEAEsepharose ${ }^{\circ} \mathrm{GE}$ healthcare, Uppsala, Sweden).

\section{Plasmodium species DNA detection by PCR}

Detection of $P$. falciparum based on the amplification of the STEVOR gene, a subtelomeric multiple copy gene, was performed according to Filisetti et al. [15]. The other infecting Plasmodium species were identified using a genus and species-specific nested PCR, in addition to a PCR tailored to a specific $P$. ovale subspecies ( $P$. ovale wallikeri) [16].

\section{ELISA using Plasmodium falciparum native antigen (NatELISA) and recombinant protein (RecELISA)}

Antibody screening was performed using an in-house ELISA test derived from a commercial assay (DiaMed) [14]. Native P. falciparum antigen produced from in vitro culture (NatELISA) [17] or recombinant rMSP1 for $P$. ovale, $P$. malariae, $P$. falciparum and $\mathrm{r} P$. falciparum AMA1 (RecELISA) were immobilized on 96-well plates overnight at $4^{\circ} \mathrm{C}$ in coating buffer and blocked for one hour with PBST (Phosphate Buffered Saline with Tween $0.05 \%$ ) containing 1\% BSA (bovine serum albumin) (Merck, Darmstadt, Germany). After washing with PBST, $200 \mu \mathrm{l}$ of diluent buffer (PBST with BSA 0.1\%) were dispensed into each well and $10 \mu \mathrm{l}$ of serum were incubated for one hour at $37^{\circ} \mathrm{C}$. On the same plate, $10 \mu \mathrm{L}$ of positive controls specific for each species and negative controls in triplicate were added. After three washes with PBST, $100 \mu \mathrm{l}$ of horseradish peroxidase labelled monoclonal rabbit anti human IgG (Sigma-Aldrich, St Quentin, France) were incubated for $30 \mathrm{~min}$ at $37^{\circ} \mathrm{C}$. After three washes with PBST, $100 \mu \mathrm{l}$ of TMB plus substrate solution (Tetramethylbenzidine) (Kem-en-tec, Denmark) were incubated for $15 \mathrm{~min}$ at $37^{\circ} \mathrm{C}$ and the reaction was stopped with $50 \mu \mathrm{l}$ of $0.5 \mathrm{M}$ sulphuric acid. The absorbance was read within $30 \mathrm{~min}$ at $450 \mathrm{~nm}$ against $620 \mathrm{~nm}$. Test validation required the positive OD to be $>0.500$ and the negative OD $<0.200$. The cut-off value was set at threefold the negative control wells' average OD. The antibody $(\mathrm{Ab})$ index of each sample was defined as ratio of its OD value and the cut-off value. The sample was considered positive if the $\mathrm{Ab}$ index was $>0.7$, and negative if the $\mathrm{Ab}$ index was $\leq 0.7$.

All recombinant assays were performed on an EVOLIS Microplate System (Bio-Rad) at the Department of Microbiology of the Hôpitaux Universitaires de Strasbourg.

\section{Statistical analysis}

Statistical differences between $P$. falciparum native antigen ELISA (NatELISA) and recombinant ELISA (RecELISA) performances were analysed using Chi-squared and t-tests, as detailed in the Results section, below. Significant differences in antibody prevalence depending on the season, observed during follow-up, were assessed using the Chi-squared test. A p-value of less than 0.05 was considered significant. Correlation between antibody titre and ELISA index for the recombinant antigens was calculated using the Pearson correlation test. (GraphPad Prism 6.0 software, La Jolla, CA, USA).

\section{Results}

\section{Performance of the RecELISAs}

The assay was first evaluated on 144 plasma samples from malaria patients diagnosed using microscopy and confirmed by PCR. The RecELISA performed better than the NatELISA, which used a $P$. falciparum native antigen (Table 1). Recombinant proteins from other Plasmodium species presented a good sensitivity; only three of 26 $P$. ovale cases were missed, and every $P$. malariae infection was detected. The combined performance of the three RecELISAs (using recombinant proteins from three species) was superior to that of the NatELISA (Table 2). On the other hand, the RecELISA failed to detect nine positive cases, most of them also missed by the NatELISA (Table 3). Three of the nine missed infections were $P$. ovale cases, while six were $P$. falciparum. However, eight cases were detected exclusively by RecELISA. Four of those were $P$. ovale and one was $P$. malariae; the three others were $P$. falciparum infections. Overall specificity was $97.9 \%$, calculated on 192 unexposed to malaria blood donors.

\section{Antibody prevalence in Beninese blood donors}

The recombinant antigens were used to evaluate species-specific antibody prevalence in a panel of Beninese blood donors. A population of blood donors with microscopically detectable Plasmodium infections was isolated from a previous study [9]. Anti-P. falciparum 
Table 1 Evaluation of the performance of ELISA using species-specific recombinant Plasmodium proteins (RecELISA) in comparison to an ELISA using $P$. falciparum native antigen (NatELISA) in a population of malaria-infected travellers ( $n=144)$ : 106 Plasmodium falciparum patients; 26 Plasmodium ovale patients and 12 Plasmodium malariae patients

\begin{tabular}{|c|c|c|c|c|c|c|}
\hline Species & $\begin{array}{l}\text { Microscopy + PCR } \\
\text { positive }\end{array}$ & NatELISA pos & $\begin{array}{l}\text { RecELISA rPfMSP1 + } \\
\text { rPfAMA1 pos }\end{array}$ & $\begin{array}{l}\text { RecELISA } \\
\text { rPoMSP1 pos }\end{array}$ & $\begin{array}{l}\text { RecELISA } \\
\text { rPmMSP1 pos }\end{array}$ & $\begin{array}{l}\text { RecELISA } \\
\text { Total pos }\end{array}$ \\
\hline P. falciparum & $106 / 144$ & $98 / 106$ (92.5\%) & $100 / 106$ & ND & ND & $135 / 144^{c}$ \\
\hline P. ovale & $26 / 144$ & 20/26 (76.9\%) & ND & $23 / 26$ & ND & \\
\hline P. malariae & $12 / 144$ & $11 / 12(91.7 \%)$ & ND & ND & $12 / 12$ & \\
\hline Negative & $N D^{b}$ & $5 / 192$ & $3 / 192$ & 3/192 & $3 / 192$ & $4 / 192$ \\
\hline
\end{tabular}

For the calculation of specificity, blood donors not exposed to malaria $(n=192)$ were used exposed to malaria $(n=192)$ were used.

aeach sample was confirmed by PCR.

${ }^{\mathrm{b}} \mathrm{ND}$ : not done. Blood donors not travelling in malaria-endemic countries were considered as negative $(n=192)$.

cresults for the combined recombinant for the three species.

antibodies were detected in almost 90\% (259/290) of the population with the NatELISA. Only two mixed infections ( $P$. malariae and P. falciparum) were detected by microscopy, confirmed by PCR, and positive with all recombinant proteins (Table 4). Combining the data from the three assays (RecELISA Total) reveals that almost all of the investigated population had antibodies against $P$. falciparum, $P$. ovale and $P$. malariae, suggesting a non-negligible presence of co-infection (Table 4). In all blood donors, $85 \%(1,050 / 1,235)$ presented antibodies directed to native P. falciparum antigen, $94.4 \%(1,166 /$ $1,235)$ to $P$. falciparum recombinant rPfMSP1 and rPfAMA1, $56.8 \%(702 / 1,235)$ to the $P$. ovale recombinant $\mathrm{rPoMSP} 1$, and $67.5 \%(834 / 1,235)$ to the $P$. malariae recombinant $\mathrm{rPmMSP} 1$. By summing up all the patients bearing antibodies directed to recombinant proteins, the prevalence was $98.8 \%(1,220 / 1,235)$ (Figure 1). These results demonstrate that the exclusive use of a $P$. falciparum native antigen leads to an underestimation of the seroprevalence of Plasmodium infection (Table 5). The 173 donors positive under RecELISA and negative under NatELISA were divided into seven populations depending on the nature of the recombinant protein detected. Notably, almost $30 \%$ of that population was positive for P. falciparum, P. ovale and P. malariae (Table 6). In the RecELISA malaria antibodies-positive population $(\mathrm{n}=1,220), 45.3 \%$ of the sera had antibodies recognizing recombinant proteins from all three species (Figure 2). According to this assay, $76.1 \%$ of the population were suspected to have an infection with at least two different species of Plasmodium.
Seroprevalence of antibodies directed to the three species throughout the four seasons of Benin's semiequatorial climate (Figure 3) was investigated. Recognition of antibodies directed to $P$. malariae and $P$. ovale rose until the end of the LDS. Finally, summing up the data from the three species, the rate of antibodies was constant throughout the year (Figure 3). Combining the three species' recombinant proteins improved sensitivity by $10 \%$ over the use of recombinant proteins from $P$. falciparum alone. The prevalence of antibodies against the native $P$. falciparum antigen dropped significantly at the SRS, and then increased at the LDS. Distribution of positive samples according to ELISA index for P. falciparum, $P$. malariae and $P$. ovale recombinant antigens was also estimated and showed low rates for antibodies directed to $P$. ovale and $P$. malariae species and higher rates for antibodies directed to P. falciparum (Figure 4). No correlation between parasite density and antibody titre for NatELISA and RecELISA for the three species was observed.

\section{Discussion}

The antigenicity of these recombinant proteins was first evaluated in patients infected by $P$. falciparum, $P$. ovale and $P$. malariae in European settings. The analytical parameters (sensitivity, specificity, PPV, and NPV) using recombinant proteins were better than those of an ELISA using a native antigen from $P$. falciparum. In particular, the use of recombinant proteins from the $P$. ovale and $P$. malariae species largely enhanced detection rates for those infections. The efficacy of these new tools was optimized using synthetic genes integrating consensus sequences

Table 2 Recapitulative performances of the assay

\begin{tabular}{llllll}
\hline Performances & NatELISA & RecELISA rPfMSP1 + rPfAMA1 & RecELISA rPoMSP1 & RecELISA rPmMSP1 & RecELISA Total \\
\hline Sensitivity & $129 / 144(89.6 \%)$ & $100 / 106(94.3 \%)$ & $23 / 26(88.5 \%)$ & $12 / 12(100 \%)$ & $135 / 144(93.8 \%)$ \\
Specificity & $187 / 192(97.4 \%)$ & $189 / 192(98.4 \%)$ & $189 / 192(98.4 \%)$ & $189 / 192(98.4 \%)$ & $188 / 192(97.9 \%)$ \\
PPV & $96.3 \%$ & $97.1 \%$ & $88.5 \%$ & $80 \%$ & $97.1 \%$ \\
NPV & $92.6 \%$ & $96.9 \%$ & $98.4 \%$ & $100 \%$ & $95.4 \%$ \\
\hline
\end{tabular}


Table 3 Performance of Plasmodium falciparum native antigen ELISA (NatELISA) versus three specific recombinant Plasmodium proteins ELISA (RecELISA Total)

\begin{tabular}{llll}
\hline & RecELISA Total Pos & RecELISA Total neg & Total \\
\hline NatELISA pos & 127 & 2 & 129 \\
NatELISA neg & 8 & 7 & 15 \\
Total & $\mathbf{1 3 5}$ & $\mathbf{9}$ & $\mathbf{1 4 4}$ \\
\hline
\end{tabular}

Total comparative results for malaria-infected patients $(n=144)$ microscopically diagnosed in a non-endemic country.

Positive RecELISA Total versus positive RecELISA: 135/144 versus 129/144: Chi-square test, $\mathrm{p}=0.2008$.

available in GenBank [4,5], covering gene polymorphisms between known strains of each species and optimized for expression in a prokaryotic system. The biobank used for validation of the reagent involved sera from patients specifically infected by one of the three species of pathogens. Specificity was high in all cases, reaching an overall level of $97.9 \%$. In general, the use of various recombinant antigens resulted in better sensitivity without sacrificing specificity. However, this technology should be further improved, especially for $P$. ovale. The surface antigen MSP1 is highly polymorphic due to wide strain variability. This pitfall in the use of recombinant proteins leading to the presentation of limited epitopes to humoral response was previously highlighted [18]. A combined analysis of more sequences from $P$. ovale strains, now accessible on GenBank, might resolve this problem.

The RecELISA's strong performance allowed the study of Beninese blood donors. Recent studies using microscopic examination in southern Benin have demonstrated the presence of mixed infection at the following rates: $2 \%$ P. ovale and $3.1 \%$ P. malariae, associated or not to $P$. falciparum: $1.2 \% P$. falciparum $/ P$. malariae, $2.4 \% P$. falciparum $/ P$. ovale and $0.1 \%$. falciparum $/ P$. malariae/P. ovale [19]. In this study, in a population of 1,235 healthy blood donors, microscopic examination revealed $21.2 \%$ P. falciparum and $0.15 \%$ P. falciparum/ P. malariae. Similarly, in Burkina Faso, microscopy detected $72.5 \%$ P. falciparum, $13.2 \%$ P. malariae and $1.8 \%$ $P$. ovale in a population of 830 children between three and 15 years old, of which $18.8 \%$ were febrile [20]. Microscopy probably underestimates prevalence, especially for the cryptic species with low parasitaemia, $P$. ovale

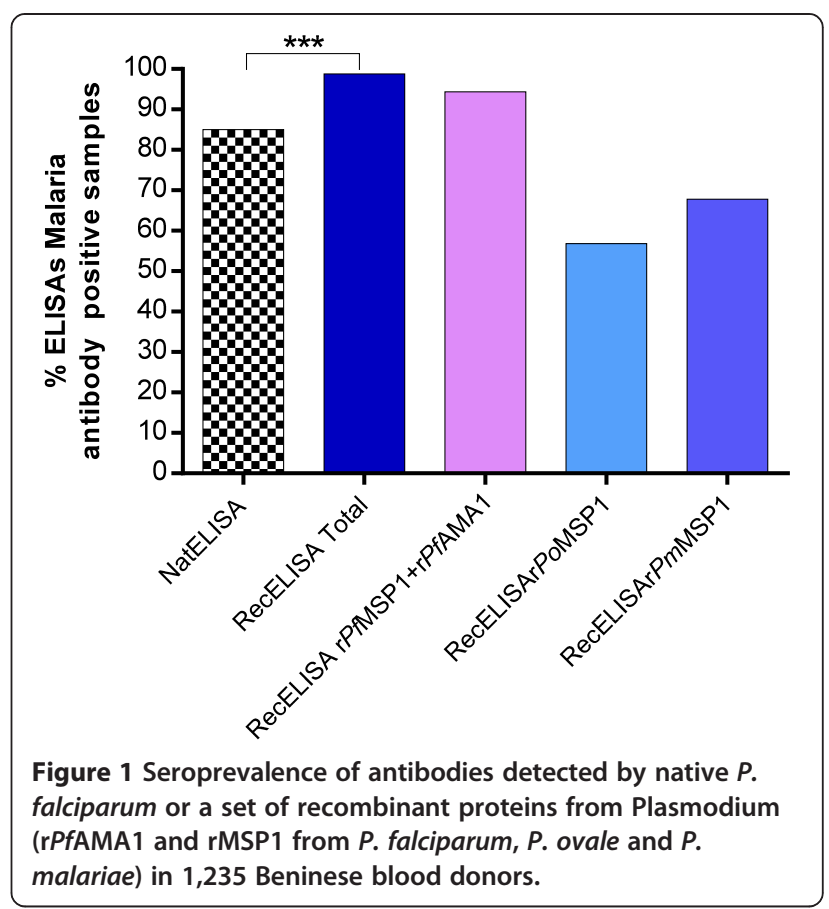

and P. malariae. This diagnostic tool has a detection threshold of five to 50 parasites/ $\mu \mathrm{L}$ [8], while PCR reaches $0.001 \mathrm{p} / \mu \mathrm{L}$ [15]. A recent review [21] showed that the use of molecular methods such as PCR, facilitates greatly increased $P$. malariae and $P$. ovale detection sensitivity. Snounou et al., using PCR, were able to detect in a microscopically negative Bissau-Guinean population, $23.3 \%$ P. malariae and $6.9 \%$ P. ovale [22] infection. However, submicroscopic and even sub-PCR infections do exist and can only be revealed with serology [11]. Therefore, delimiting transmission areas is essential [10]. Thus, seroprevalence reflects exposure, overcoming sampling bias associated with seasonal and year-to-year variation [11].

However, seroprevalence was exclusively tested using $P$. falciparum antigens, neglecting $P$. ovale and $P$. malariae. The use of recombinant proteins of $P$. malariae and $P$. ovale allowed revealing high prevalence of antibodies directed to these cryptic parasites. In Benin, an increased production of antibodies directed to $P$. ovale and P. malariae was observed until the LDS. This may be

Table 4 Evaluation of the seroprevalence of three Plasmodium species in blood donors from Benin by the three specific recombinant Plasmodium proteins ELISA (RecELISA) and Plasmodium falciparum native antigen ELISA (NatELISA) in a population of asymptomatic Benin blood donors with positive parasitaemia ( $\mathrm{n}=290$ ): 288 Plasmodium falciparum and two mixed infection Plasmodium falciparum and Plasmodium malariae identified by microscopy

\begin{tabular}{|c|c|c|c|c|c|}
\hline Results of microscopical examination & NatELISA & RecELISA rPfMSP1 + rPfAMA1 & $\begin{array}{l}\text { RecELISA } \\
\text { rPoMSP1 }\end{array}$ & $\begin{array}{l}\text { RecELISA } \\
\text { rPmMSP1 }\end{array}$ & $\begin{array}{l}\text { RecELISA } \\
\text { Total }\end{array}$ \\
\hline 288 P. falciparum & $257 / 288(89.2 \%)$ & $282 / 288(97.9 \%)$ & $195 / 288(67.7 \%)$ & $229 / 288(79.9 \%)$ & $287 / 288(99.6 \%)$ \\
\hline 2 P. malariae and P. falciparum & $2 / 2(100 \%)$ & $2 / 2(100 \%)$ & $2 / 2(100 \%)$ & $2 / 2(100 \%)$ & $2 / 2(100 \%)$ \\
\hline
\end{tabular}


Table 5 Comparative results of Plasmodium falciparum native antigen ELISA (NatELISA) and three specific recombinant Plasmodium proteins ELISA (RecELISA Total) for Beninese blood donors $(n=1,235)$

\begin{tabular}{llll}
\hline & RecELISA Total Pos & RecELISA Total Neg & Total \\
\hline NatELISA pos & 1047 & 3 & 1050 \\
NatELISA neg & 173 & 12 & 185 \\
Total & $\mathbf{1 2 2 0}$ & $\mathbf{1 5}$ & $\mathbf{1 2 3 5}$ \\
\hline
\end{tabular}

Positive RecELISA Total versus positive NatELISA: 1,220/1,235 versus 1,050/1,235: Chi-square test, $p<0.0001$.

due to the delayed appearance of these species after the first infection, as has already been observed [23]. Using recombinant proteins from $P$. falciparum (MSP1 and AMA1), seroprevalence increased after the LRS and maintained high levels until the LDS, reflecting the longlasting presence of IgG directed to rPfAMA1 and rPfMSP1 antigens. Similarly, the seroprevalence against $P$. falciparum recombinant proteins did not vary between wet and dry seasons in the Gambia [24]. Interestingly, a drop in the recognition of a native $P$. falciparum antigen was observed during the SRS. This may be due to a deficit of MSP1 and AMA1 in the native antigen, prepared from a synchronized culture in which an $80 \%$ schizont proportion shifted the recognition of the malarial antibodies towards the detection of older stages of $P$. falciparum. Another hypothesis is that the variation in antibody response is due to differences in antigen concentration and relative proportions of MSP1 in native and recombinant antigenic preparations.

This work shows that more than half of the studied population was in contact with other species of Plasmodium in addition to P. falciparum, and that $76 \%$ of the investigated healthy population were exposed to more than one species. These results indicate that the prevalence of non-falciparum species is much higher than previously estimated. The levels of antibodies directed to

Table 6 Distribution of species in the population negative for the Plasmodium falciparum native antigen ELISA (NatELISA) and positive for the three specific recombinant Plasmodium proteins ELISA (RecELISA Total)

\begin{tabular}{lll}
\hline Species & RecELISATotal pos & Percentage \\
\hline $\boldsymbol{P f}$ & 65 & $37.6 \%$ \\
Pm & 6 & $3.5 \%$ \\
Po & 3 & $1.7 \%$ \\
Po + Pf & 20 & $11.6 \%$ \\
$P \boldsymbol{P}+\boldsymbol{P m}$ & 3 & $1.7 \%$ \\
$\boldsymbol{P m}+\boldsymbol{P f}$ & 28 & $16.2 \%$ \\
$\boldsymbol{P f}+\boldsymbol{P o}+\boldsymbol{P m}$ & 48 & $\mathbf{2 7 . 7 \%}$ \\
Total & 173 & \\
\hline
\end{tabular}

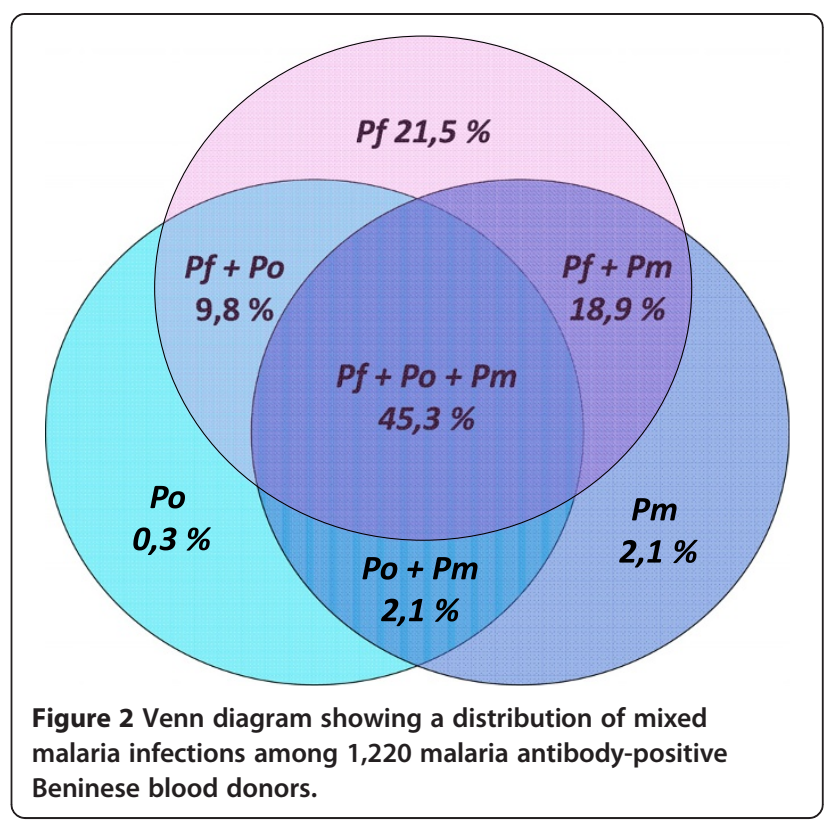

these two cryptic species is low, possibly due to their parasitaemia levels. On the contrary, high levels of antibodies against $P$. falciparum are observed, corresponding to high parasitaemia levels.

While $P$. falciparum infection remains the primary concern, the detection of $P$. ovale and P. malariae is still important. Although both cause mild infection, $P$. malariae can cause chronic nephritic syndrome, leading to adverse reactions during treatment and a high rate of mortality

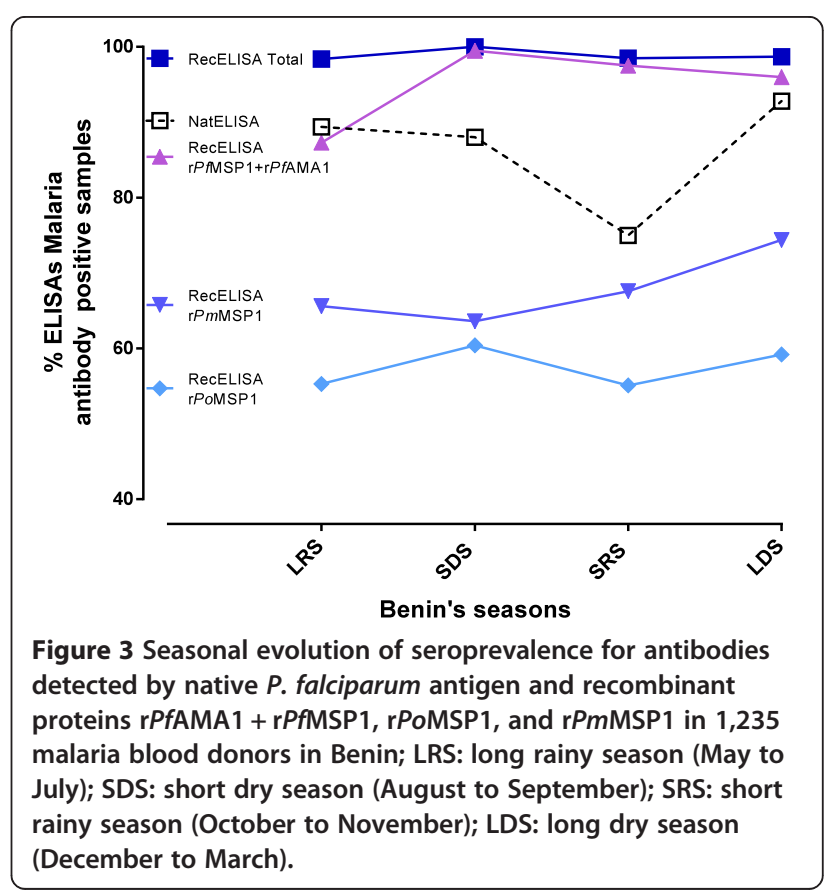




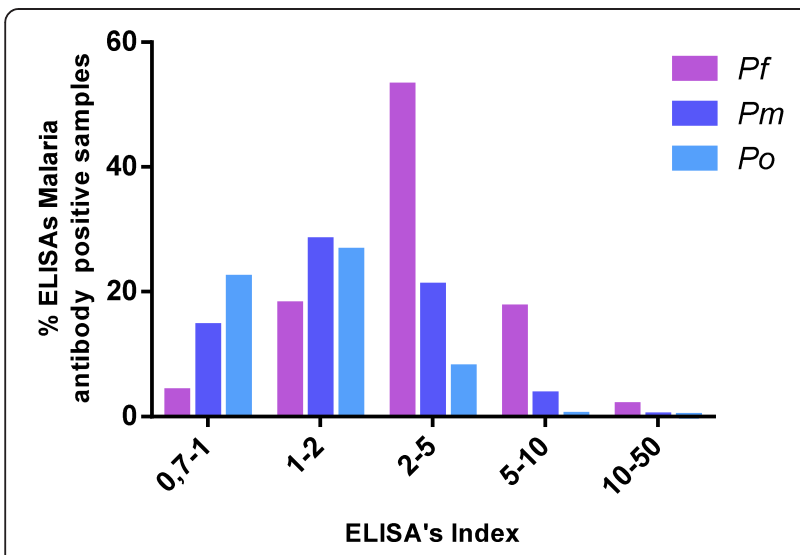

Figure 4 Distribution of positive samples according to the ELISA's index for $P$. falciparum, $P$. malaria and $P$. ovale recombinant antigens. The cut-off value was calculated by multiplying the negative control wells' average optical density (OD) by three. The antibody (Ab) index of each sample was calculated by dividing its OD value by the cut-off value. The sample was considered positive if the $A b$ index was $>0.7$, and negative if the $A b$ index was $\leq 0.7$.

[25]. There is also a risk of recurrence decades after initial exposure, even when the infected population has left the endemic region [26]. Immune protection against $P$. falciparum is not fully effective against $P$. malariae [27]. Furthermore, P. malariae is more prevalent when transmission and infection rates of $P$. falciparum are lower, probably because of negative interactions with $P$. falciparum; similar interactions with $P$. ovale might exist [28]. Positive selection of anti-P. falciparum medication caused the prevalence of $P$. malariae to rise for four years in Burkina Faso. The authors predicted that in the absence of $P$. falciparum (ie, due to the effects of a vaccine directed against $P$. falciparum), P. malariae parasitaemia and gametocyte carriage could increase and maintain severe malaria infection [20].

\section{Conclusion}

Given the advent of vaccine campaigns against $P$. falciparum, other species must be monitored more closely as their presence will remain a serious health concern for exposed populations in endemic countries [29].

In conclusion, with the elimination of malaria back on the agendas of world agencies, it is necessary to monitor the effects of anti-malarial measures. In this work a standardized, sensitive and specific tool was developed that facilitates measuring and monitoring the transmission intensity of three Plasmodium species. Antibody prevalence for all species of Plasmodium will permit detection of residual infections and variations in malaria transmission. Of course, more complex combinations of recombinant Plasmodium proteins should be used in future studies to avoid the disadvantages of antigenic polymorphism and variable individual responsiveness, especially for $P$. ovale. These kinds of recombinant tools could also be used to enhance blood transmission safety in non-endemic regions [30].

\section{Competing interests}

The authors declare that they have no competing interests.

\section{Authors' contributions}

$\mathrm{CDL}, \mathrm{PA}, \mathrm{AS}$, and EC designed the study, analysed the data and prepared the report; $A S$ and EC coordinated the study; $C D L$ and PA led the study in each site; $L M$ produced the recombinant proteins; $E H, S P, D F, A A, A W P$, and JB processed the samples and collected the data in France; NWC, CDA and LA collected the data in Africa. All authors contributed to correcting the report. All authors read and approved the final manuscript.

\section{Acknowledgements}

We would like to thank technicians Rachel Huber and Sylvie Mattern from the Department of Microbiology at the Hôpitaux Universitaires de Strasbourg, for technical expertise.

\section{Author details}

${ }^{1}$ Institut de Parasitologie et de Pathologie Tropicale Université de Strasbourg, 3, Rue Koeberlé, F67000 Strasbourg, France. 'Laboratoire de Biochimie et de Biologie Moléculaire, Université d'Abomey Calavi, 04 BP 0320 Cotonou, Bénin. ${ }^{3}$ Agence Nationale pour la Transfusion Sanguine (Ministère de la Santé), 01 B.P. 511 Cotonou, Bénin.

Received: 21 March 2014 Accepted: 29 May 2014 Published: 18 June 2014

\section{References}

1. WHO: World Malaria Report 2012. Geneva: World Health Organization; 2012.

2. Greenwood BM, Bojang K, Whitty CJ, Targett GA: Malaria. Lancet 2005, 365:1487-1498.

3. Moorthy VS, Good MF, Hill AV: Malaria vaccine developments. Lancet 2004, 363:150-156.

4. Birkenmeyer L, Muerhoff AS, Dawson GJ, Desai SM: Isolation and characterization of the MSP1 genes from Plasmodium malariae and Plasmodium ovale. Am J Trop Med Hyg 2010, 82:996-1003.

5. Muerhoff AS, Birkenmeyer LG, Coffey R, Dille BJ, Barnwell JW, Collins WE, Sullivan JS, Dawson GJ, Desai SM: Detection of Plasmodium falciparum, $P$. vivax, $P$. ovale, and $P$. malariae merozoite surface protein 1-p19 antibodies in human malaria patients and experimentally infected nonhuman primates. Clin Vaccine Immunol 2010, 17:1631-1638.

6. Sachdeva S, Mohmmed A, Dasaradhi PV, Crabb BS, Katyal A, Malhotra P, Chauhan VS: Immunogenicity and protective efficacy of Escherichia coli expressed Plasmodium falciparum merozoite surface protein-1(42) using human compatible adjuvants. Vaccine 2006, 24:2007-2016.

7. Courtin D, Oesterholt M, Huismans H, Kusi K, Milet J, Badaut C, Gaye O, Roeffen W, Remarque EJ, Sauerwein R, Garcia A, Luty AJ: The quantity and quality of African children's $\lg G$ responses to merozoite surface antigens reflect protection against Plasmodium falciparum malaria. PLoS One 2009, 4:e7590.

8. Moody A: Rapid diagnostic tests for malaria parasites. Clin Microbiol Rev 2002, 15:66-78.

9. Atchade PS, Doderer-Lang C, Chabi N, Perrotey S, Abdelrahman T, Akpovi CD, Anani L, Bigot A, Sanni A, Candolfi E: Is a Plasmodium lactate dehydrogenase (pLDH) enzyme-linked immunosorbent (ELISA)-based assay a valid tool for detecting risky malaria blood donations in Africa? Malar J 2013, 12:279.

10. Drakeley C, Cook J, Rollinson D, Hay SI: Potential contribution of seroepidemiological analysis for monitoring malaria control and elimination: historical and current perspectives. Adv Parasitol 2009, 69:299-352.

11. Drakeley CJ, Corran PH, Coleman PG, Tongren JE, MCDonald SL, Carneiro I, Malima R, Lusingu J, Manjurano A, Nkya WM, Lemnge MM, Cox J, Reyburn H, Riley EM: Estimating medium- and long-term trends in malaria transmission by using serological markers of malaria exposure. Proc Natl Acad Sci U S A 2005, 102:5108-5113. 
12. Druilhe P, Pradier O, Marc JP, Miltgen F, Mazier D, Parent G: Levels of antibodies to Plasmodium falciparum sporozoite surface antigens reflect malaria transmission rates and are persistent in the absence of reinfection. Infect Immun 1986, 53:393-397.

13. Seed CR, Kitchen A, Davis TM: The current status and potential role of laboratory testing to prevent transfusion-transmitted malaria. Transfus Med Rev 2005, 19:229-240.

14. Doderer $C$, Heschung A, Guntz P, Cazenave JP, Hansmann Y, Senegas A Pfaff AW, Abelrahman T, Candolfi E: A new ELISA kit which uses a combination of $P$. falciparum extract and recombinant $P$. vivax antigens as an alternative to IFAT for detection of malaria antibodies. Malar $J$ 2007, 6:19.

15. Filisetti D, Bombard S, N'Guiri C, Dahan R, Molet B, Abou-Bacar A, Hansmann Y, Christmann D, Candolfi E: Prospective assessment of a new polymerase chain reaction target (STEVOR) for imported Plasmodium falciparum malaria. Eur J Clin Microbiol Infect Dis 2002, 21:679-681.

16. Calderaro A, Piccolo G, Perandin F, Gorrini C, Peruzzi S, Zuelli C, Ricci L, Manca N, Dettori G, Chezzi C, Snounou G: Genetic polymorphisms influence Plasmodium ovale PCR detection accuracy. J Clin Microbiol 2007, 45:1624-1627.

17. Trager W, Jensen JB: Human malaria parasites in continuous culture. Science 1976, 193:673-675.

18. Taylor RR, Egan A, McGuinness D, Jepson A, Adair R, Drakely C, Riley E: Selective recognition of malaria antigens by human serum antibodies is not genetically determined but demonstrates some features of clonal imprinting. Int Immunol 1996, 8:905-915.

19. Damien GB, Djenontin A, Rogier C, Corbel V, Bangana SB, Chandre F, Akogbeto M, Kinde-Gazard D, Massougbodji A, Henry MC: Malaria infection and disease in an area with pyrethroid-resistant vectors in southern Benin. Malar J 2010, 9:380.

20. Gneme A, Guelbeogo WM, Riehle MM, Tiono AB, Diarra A, Kabre GB, Sagnon N, Vernick KD: Plasmodium species occurrence, temporal distribution and interaction in a child-aged population in rural Burkina Faso. Malar J 2013, 12:67.

21. Mueller I, Zimmerman PA, Reeder JC: Plasmodium malariae and Plasmodium ovale "the bashfull" malaria parasites. Trends Parasitol 2007 23:278-283.

22. Snounou G, Pinheiro L, Goncalves A, Fonseca L, Dias F, Brown KN, do Rosario VE: The importance of sensitive detection of malaria parasites in the human and insect hosts in epidemiological studies, as shown by the analysis of field samples from Guinea Bissau. Trans $R$ Soc Trop Med Hyg 1993, 87:649-653.

23. Molineaux L, Storey J, Cohen JE, Thomas A: A longitudinal study of human malaria in the West African Savanna in the absence of control measures: relationships between different Plasmodium species, in particular P. falciparum and P. malariae. Am J Trop Med Hyg 1980, 29:725-737.

24. Oduro AR, Conway DJ, Schellenberg D, Satoguina J, Greenwood BM, Bojang KA: Seroepidemiological and parasitological evaluation of the heterogeneity of malaria infection in the Gambia. Malar J 2013, 12:222

25. Eiam-Ong S: Malarial nephropathy. Semin Nephrol 2003, 23:21-33.

26. Siala E, Khalfaoui M, Bouratbine A, Hamdi S, Hili K, Aoun K: Relapse of Plasmodium malariae malaria 20 years after living in an endemic area (in French). Presse Med 2005, 34:371-372.

27. Boudin C, Robert V, Verhave JP, Carnevale P, Ambroise-Thomas P: Plasmodium falciparum and $P$. malariae epidemiology in a West African village. Bull World Health Organ 1991, 69:199-205.

28. Mason DP, McKenzie FE, Bossert WH: The blood-stage dynamics of mixed Plasmodium malariae-Plasmodium falciparum infections. J Theor Biol 1999, 198:549-566.

29. Agnandji ST, Lell B, Soulanoudjingar SS, Fernandes JF, Abossolo BP, Conzelmann C, Methogo BG, Doucka Y, Flamen A, Mordmuller B, Issifou S, Kremsner PG, Sacarlal J, Aide P, Lanaspa M, Aponte JJ, Nhamuave A Quelhas D, Bassat Q, Mandjate S, Macete E, Alonso P, Abdulla S, Salim N, Juma O, Shomari M, Shubis K, Machera F, Hamad AS, Minja R, et al: First results of phase 3 trial of RTS, S/AS01 malaria vaccine in African children. N Engl J Med 2011, 365:1863-1875.
30. Reesink HW, Panzer S, Wendel S, Levi JE, Ullum H, Ekblom-Kullberg $S$, Seifried E, Schmidt M, Shinar E, Prati D, Berzuini A, Ghosh S, Flesland O, Jeansson S, Zhiburt E, Piron M, Sauleda S, Ekermo B, Eglin R, Kitchen A, Dodd RY, Leiby DA, Katz LM, Kleinman S: The use of malaria antibody tests in the prevention of transfusion-transmitted malaria. Vox Sang 2011, 98:468-478.

doi:10.1186/1475-2875-13-240

Cite this article as: Doderer-Lang et al:: The ears of the African elephant: unexpected high seroprevalence of Plasmodium ovale and Plasmodium malariae in healthy populations in Western Africa. Malaria Journal 2014 13:240

\section{Submit your next manuscript to BioMed Central and take full advantage of:}

- Convenient online submission

- Thorough peer review

- No space constraints or color figure charges

- Immediate publication on acceptance

- Inclusion in PubMed, CAS, Scopus and Google Scholar

- Research which is freely available for redistribution

Submit your manuscript at www.biomedcentral.com/submit
C Biomed Central 